\title{
String-inspired higher-curvature terms and the Randall-Sundrum scenario
}

\author{
Nick E. Mavromatos \\ Department of Physics, Theoretical Physics, King's College London, Strand, London WC2R 2LS, United Kingdom \\ John Rizos \\ Department of Physics, University of Ioannina, GR 45100 Ioannina, Greece \\ and CERN, European Organization for Nuclear Research, Theory Division, CH-1211 Geneva 23, Switzerland
}

(Received 14 August 2000; published 16 November 2000)

\begin{abstract}
We consider the $O\left(\alpha^{\prime}\right)$ string effective action, with Gauss-Bonnet curvature-squared and fourth-order dilaton-derivative terms, which is derived by a matching procedure with string amplitudes in five space-time dimensions. We show that a non-factorizable metric of the Randall-Sundrum (RS) type, with a fourdimensional conformal factor $e^{-2 k|z|}$, can be a solution of the pertinent equations of motion. The parameter $k$ is found to be proportional to the string coupling $g_{s}$ and thus the solution appears to be non-perturbative. It is crucial that the Gauss-Bonnet combination have the right (positive in our conventions) sign, relative to the Einstein term, which is the case necessitated by compatibility with string (tree) amplitude computations. We study the general solution for the dilaton and metric functions, and thus construct the appropriate phase-space diagram in the solution space. In the case of an anti-de Sitter bulk, we demonstrate that there exists a continuous interpolation between (part of) the RS solution at $z=+\infty$ and an (integrable) naked singularity at $z=0$. This implies the dynamical formation of domain walls (separated by an infinite distance), thus restricting the physical bulk space-time to the positive $z$ axis. Some brief comments on the possibility of fine-tuning the four-dimensional cosmological constant to zero are also presented.

PACS number(s): 04.50.+h, 11.10.Kk, 98.80.Cq
\end{abstract}

\section{INTRODUCTION}

Recently considerable effort has been devoted to the study of higher-dimensional space-times with metrics of nonfactorizable form between four- and higher- (bulk) dimensional coordinates $[1,2]$ :

$$
d s^{2}=e^{-2 \sigma(z)} \eta_{i j} d X^{i} d X^{j}+d z^{2}, \quad i, j=0,1, \ldots, 3
$$

In the modern context of non-perturbative string (brane) theory, this type of metrics arises from the so-called $\mathrm{D}$ (irichlet)-brane picture of our world, according to which the observable Universe is viewed as a three-brane embedded in a higher-dimensional (bulk) geometry [3,2]. Among other issues, in such an approach one looks for mechanisms that solve the mass hierarchy problem [2] or offer explanations for the vanishing of the (four-dimensional) cosmological constant. However, the latter case is inflicted by the presence of naked singularities in the bulk [4] and/or instabilities $[5]$.

In the original approach $[1,2]$ the metric (1) has been considered only in connection with Einstein-type theories of gravitation, i.e. theories in which only the curvature scalar appears in the gravitational part of the action. Recently, however, attempts have been made towards the inclusion of higher-curvature (quadratic) terms in the action [6] of the Gauss-Bonnet (GB) type [7]. Such terms, which arise naturally in (super)string effective actions [8], are known to lead to non-trivial cosmological and general-relativistic solutions, such as singularity-free expanding [9] and/or closed [10] universes, and black-hole solutions with non-trivial (secondary dilaton) hair $[11,12]$.
In Ref. [6] five-dimensional bulk geometries were considered, with our four-dimensional universe viewed as a threebrane embedded in them. It was argued, in agreement with the lowest-order (in the scalar curvature) results [4], that the presence of higher-curvature Gauss-Bonnet terms cannot lead to a solution of the cosmological constant on the brane without fine-tuning, as a result of the appearance of naked singularities in the bulk. However, in the models considered in Ref. [6], the Gauss-Bonnet term in the action was decoupled from the dilaton field. This is not the case in stringeffective models of higher-derivative gravity, compatible with string (tree) amplitude computations in the bulk geometry [8]. In the latter case, it is known that the dilaton field $\Phi$ couples to the higher-curvature part of the effective action through the appropriate conformal weight, $e^{m \Phi}$. The weight $m$ is determined, together with the coefficient of the GB terms, by the requirement that the effective action be the one reproduced by the appropriate string amplitudes [8].

The purpose of this work is to reconsider the solution under the inclusion of proper string-effective highercurvature terms. In this article we show that, in a setup where there is an initial three-dimensional (spatial) brane located at the origin $z=0$ of the bulk dimension of the five-dimensional geometry, a metric of the form (1) is still a solution of the equations of motion of an effective action derived from (conventional) string amplitudes [8], up to $O\left(\alpha^{\prime}\right)$ in the Regge slope $\alpha^{\prime}$. As is well known, such actions can always be cast, by means of appropriate field redefinitions that leave the (perturbative) string amplitudes invariant, in a GB form [7], provided one includes appropriate fourth-derivative dilaton terms. In fact, as we shall show below, both of these facts result in different conclusions, for the non-constant dilaton case, from those in Ref. [6]. 
It is important to note that the sign, as well as the relative strength $\lambda$ of the GB terms in the action, is uniquely determined by the amplitude-matching procedure. In our conventions for the metric and curvature the coefficient $\lambda$ comes out positive. We shall demonstrate that the Randall-Sundrum (RS) type metric [2], with

$$
\sigma(z)=\sum_{i} k\left|z-z_{i}\right|
$$

where $i$ denotes the $i$ th brane, located at $z_{i}$ along the bulk direction, satisfies the equations of motion derived from such an $O\left(\alpha^{\prime}\right)$ string-effective action. It is important to stress that the solution exists only for $\lambda>0$. Moreover, the parameter $k \propto \sqrt{1 / \lambda}$. Since [8] $\lambda \propto 1 / g_{s}^{2}$, where $g_{s}$ is the string coupling, the resulting solution appears (formally) non-perturbative.

As we shall show, in our scenario there are also solutions that are characterized by a vanishing vacuum energy contribution on the brane, a requirement that may come, for instance, by demanding a consistent embedding of the solution (1) in a supersymmetric theory on the brane. However, as argued in [13], recoil (quantum) fluctuations of the D3brane, as a result of scattering with (bulk) closed strings or other solitonic defects, may induce supersymmetry obstruction by means of "conical" singularities on the brane. This yields small contributions to the vacuum energy of the brane, which, as a result of recoil, finds itself in an excited state, rather than its ground state. In addition, recoil fluctuations lead to a dynamical formation of horizons in the bulk dimension [14] of a given size, which is determined by the dynamics. Such effects, which here are viewed as subleading to the classical ones we are discussing, will be the topic of a forthcoming publication.

In the present article we shall consider dilaton configurations that depend solely on the bulk dimension $z$. A particularly interesting case is the one in which the dilaton field is linear in $z$. This case may be motivated by the fact that the equations of motion of fields in the geometry (1) acquire a "friction type" form, suggestive of the role of the bulk dimension as a renormalization group (RG) parameter [15] and, actually, of the Liouville-field type [16-18]. The spacelike character of the Liouville field is dictated by the subcritical dimensionality of space-time in the specific fivedimensional geometry under consideration. Crucial to this interpretation is the fact that the bulk space-time is of antide-Sitter type, which is known to exhibit holographic properties [19]. The fact that there exist non-trivial solutions to the equations of motion, including the (stringy) GaussBonnet term, is suggestive of a deeper connection of this string-inspired approach with the (holographic) bulk geometries (1). However, in this paper we shall not pursue the holographic RG interpretation in detail. We only mention at this stage that this interpretation does not seem to hold in the generic case, and requires specific properties of the bulk geometry (e.g. the validity of a proper $c$ theorem [20-22]), which could be quite restrictive in the presence of highercurvature terms. A detailed study of such important issues will be the topic of a forthcoming publication.
The structure of the article is as follows: in Sec. II we formulate the problem, and discuss the GB higher-curvature combination and its connection with string amplitudes in a general context of a $D$-dimensional space-time. In Sec. III we discuss non-factorizable metrics of the form (1) in a fivedimensional setup, with the fifth dimension generating a bulk geometry, in which a three-brane world is embedded. In particular, we first demonstrate the consistency of the RandallSundrum-type space-time with a constant dilaton, in the presence of the Gauss-Bonnet higher-curvature combination derived from string amplitudes. We then proceed to discussing the linear-dilaton ansatz (with respect to the bulk coordinate $z$ ). We show that the string amplitude induced GaussBonnet combination is not consistent with this solution. However, there is a solution corresponding to a case [6] in which there is no conformal coupling of the dilaton with the higher-curvature terms in the effective action. Our solution, however, still differs from that of Ref. [6] because of the presence of fourth-derivative dilaton terms. Some brief comments on the possibility of fine-tuning the four-dimensional cosmological constant to zero are made. In particular, our analysis demonstrates that such fine-tuning is possible only in the constant dilaton case. In Sec. IV, we discuss the general solution of the equations of motion for the dilaton and graviton fields in the string-effective case. This includes the above solutions as special cases. In this general case, one is capable of presenting some analytic arguments on the singularity structure of the solutions, which allow important conclusions to be drawn on the underlying physics, which go beyond the numerical solutions obtained. In particular, in the string amplitude effective case, we demonstrate the existence of new solutions consisting of continuous functions for the dilaton and space-time metric fields that interpolate between a RS-type solution at $z=+\infty$ and an (integrable) naked singularity at $z=0$. This implies the dynamical formation of domain walls in the bulk geometry obtained from the stringeffective action. The walls are separated by an infinite distance, and this results in a dynamical restriction of the physical bulk space-time on the positive $z$ axis only. The fact that this solution emerges from (perturbative) string-effective actions is remarkable in our opinion, implying that perturbative world-sheet physics can still lead to important conclusions of relevance to (non-perturbative) string theory. Some conclusions and outlook are presented in Sec. V.

\section{STRING AMPLITUDE-INDUCED HIGHER-CURVATURE GRAVITY}

In this section we shall formulate the problem mathematically, and set up our notation and conventions. Throughout this work we shall follow the conventions of Ref. [23], according to which the five-dimensional space-time has signature $(-,+, \ldots,+)$, and the Riemann tensor is defined as $R_{\mu \nu \sigma}^{\tau}=\Gamma_{\nu \sigma, \mu}^{\tau}-\cdots$.

We consider the action

$$
S=S_{5}+S_{4}
$$

where $S_{5}$ is the five-dimensional part: 


$$
\begin{aligned}
S_{5}= & \int d^{5} x \sqrt{-g}\left[-R-\frac{4}{3}\left(\nabla_{\mu} \Phi\right)^{2}+f(\Phi)\left(\alpha R^{2}+\beta R_{\mu \nu}^{2}\right.\right. \\
& \left.\left.+\gamma R_{\mu \nu \rho \sigma}^{2}\right)+\xi(z) e^{\zeta \Phi}+c_{2} f(\Phi)\left(\nabla_{\mu} \Phi\right)^{4}+\cdots\right],
\end{aligned}
$$

with $\Phi$ the dilaton field, and the ellipsis denoting other types of contraction of the four-derivative dilaton terms; these will not be of interest to us here, for reasons that will be explained below.

The four-dimensional part $S_{4}$ of the action (3) is defined as

$$
S_{4}=\sum_{i} \int d^{4} x \sqrt{-g_{(4)}} e^{\omega \Phi} v\left(z_{i}\right)
$$

where

$$
g_{(4)}^{\mu \nu}=\left\{\begin{array}{l}
g^{\mu \nu}, \quad \mu, \nu<5, \\
0 \quad \text { otherwise, }
\end{array}\right.
$$

and the sum over $i$ extends over D-brane walls located at $z$ $=z_{i}$ along the fifth dimension. ${ }^{1}$

The quantities $\alpha, \beta, \gamma, c_{2}$ are constants to be determined below by matching with string amplitudes in the bulk geometry. We notice that in our approach we consider the vacuum energy in the bulk and on the brane as having a specific (exponential) dependence on the dilaton field $\Phi$, dictated by string amplitude computations. More general models, in which one considers arbitrary scalar potential functions of $\Phi$, have also been considered in the literature [24], but will not be analyzed here. We simply mention that the precise dynamics behind models with dilaton potentials is still unknown; in tree-level critical string theory there are no such potentials, but string-loop corrections may be responsible for their generation.

We now consider, for definiteness, the case in which the action $S_{5}$ is derived from a $O\left(\alpha^{\prime}\right)$ ( $\alpha^{\prime}$ the Regge slope) heterotic-type string theory in the low-energy limit in $D$ $(=5)$ space-time dimensions. Some remarks are in order at this point. From a formal point of view, one may think of the (bulk) fifth dimension in the space-time (1) as a (space-like) Liouville mode $[15,16]$. A more conventional (and probably safer) approach, which we shall adopt here, is to assume initially a ten-dimensional space-time, in which three branes are embedded. In the bulk one may, then, consider the propagation of closed strings only [3], but take the case in which all but one of the bulk coordinates are compactified. In that case, the induced string theory amplitudes will formally correspond to those residing in an effective 5-dimensional space-time, in the sense that one may consider string backgrounds that depend only on the uncompactified coordinates, and restrict oneself to effective string amplitudes (or, equivalently, $\sigma$-model conformal-invariance conditions [8]) for those degrees of freedom.

With the above in mind, we have [8]

$$
\alpha=+1, \quad f(\Phi)=\lambda e^{\theta \Phi}, \quad \lambda=\alpha^{\prime} / 8 g_{s}^{2}>0,
$$

where $g_{s}$ is the string coupling. In this case we also have $\zeta$ $=-\theta=4 / \sqrt{3(D-2)}[=4 / 3$ in $D=5$ dimensions of (formal) interest to us here]. Moreover, in (perturbative) string theory one has the freedom [8] to redefine the graviton and dilaton fields so as to ensure that the quadratic-curvature terms in Eq. (4) are of the ghost-free GB form [7]:

$$
\mathcal{R}_{G B}^{2}=R_{\mu \nu \rho \sigma} R^{\mu \nu \rho \sigma}-4 R_{\mu \nu} R^{\mu \nu}+R^{2} .
$$

This field-redefinition ambiguity also allows us to consider the four-derivative dilaton terms in Eq. (4) as having the single structure exhibited above. Matching with tree-level string amplitudes to $O\left(\alpha^{\prime}\right)$ then requires [8]

$$
c_{2}=\frac{16}{9} \frac{D-4}{D-2} \text {. }
$$

It is interesting to note that for four dimensional targets, this coefficient vanishes. This fourth-derivative dilaton term will turn out to yield, in the five-dimensional case, the essential difference in the solutions obtained here from those in Ref. [6].

The graviton equations of motion derived from Eq. (4) in the effective string case are (with $\alpha=\gamma=1, \beta=-4, c_{2}$ $=16 / 27$ )

$$
\begin{aligned}
0= & R^{\mu \nu}+\frac{1}{2} g^{\mu \nu}\left(-R-\frac{4}{3}(\nabla \Phi)^{2}+c_{2} f(\Phi)(\nabla \Phi)^{4}+\xi(z) e^{\zeta \Phi}\right)+\frac{1}{2} \sum_{i} \frac{\sqrt{-g_{(4)}}}{\sqrt{-g}} g_{(4)}^{\mu \nu} e^{\omega \Phi} v\left(z_{i}\right)-\frac{4}{3}\left(\nabla^{\mu} \Phi\right)\left(\nabla^{\nu} \Phi\right)-f(\Phi) \\
& \times\left(2 \alpha R R^{\mu \nu}+2 \beta R^{\mu}{ }_{\sigma} R^{\nu \sigma}+2 \gamma R_{\sigma \tau \rho}^{\mu} R^{\nu \sigma \tau \rho}\right)+\frac{1}{2} g^{\mu \nu} f(\Phi)\left(\alpha R^{2}+\beta R_{\sigma \tau} R^{\sigma \tau}+\gamma R_{\sigma \tau \rho k} R^{\sigma \tau \rho k}\right)+2 \alpha\left\{\left(g^{\mu \nu} f(\Phi) R\right)_{; \sigma}{ }^{\sigma}\right. \\
& \left.-(f(\Phi) R)_{;}^{\mu \nu}\right\}+\beta\left\{\left(g^{\mu \nu} f(\Phi) R^{\sigma \tau}\right)_{; \sigma \tau}+\left(f(\Phi) R^{\mu \nu}\right)_{; \sigma}{ }^{\sigma}-\left(f(\Phi) R^{\mu \sigma}\right)_{; \sigma}^{\nu}-\left(f(\Phi) R^{\nu \sigma}\right)_{; \sigma \sigma}^{\mu}\right\}+2 \gamma\left\{\left(f(\Phi) R^{\mu \sigma \nu \tau}\right)_{; \sigma \tau}\right. \\
& \left.+\left(f(\Phi) R^{\mu \sigma \nu \tau}\right)_{; \tau \sigma}\right\}-2 c_{2} f(\Phi)\left(\nabla^{\mu} \Phi\right)\left(\nabla^{\nu} \Phi\right)(\nabla \Phi)^{2}
\end{aligned}
$$

\footnotetext{
${ }^{1}$ It is also possible to consider [14] a "stuck" of such D-branes, in which case $\Sigma_{i}$ is replaced by $\int d z$ over flat integration measure, and $v\left(z_{i}\right) \rightarrow v(z)$. This term is not varied with respect to the fifth dimensional (bulk) gravitational field.
} 
where a semicolon denotes covariant differentiation.

The dilaton equation of motion, on the other hand, yields

$$
\begin{aligned}
0= & \frac{8}{3} \nabla^{2} \Phi+f^{\prime}(\Phi)\left(\alpha R^{2}+\beta R_{\mu \nu} R^{\mu \nu}+\gamma R_{\mu \nu \rho \sigma} R^{\mu \nu \rho \sigma}\right) \\
& +\sum_{i} \frac{\sqrt{-g_{(4)}}}{\sqrt{-g}} \omega e^{\omega \Phi} v\left(z_{i}\right)-4 c_{2} \nabla_{\mu}\left(f(\Phi)\left(\nabla^{\mu} \Phi\right)(\nabla \Phi)^{2}\right) \\
& +\zeta \xi(z) e^{\zeta \Phi}+c_{2} f^{\prime}(\Phi)(\nabla \Phi)^{4},
\end{aligned}
$$

where the prime denotes differentiation with respect to $\Phi$.

In the next two sections we shall study the classical solutions of these equations in the context of non-factorizable space-times of the form (1).

\section{STRING-INDUCED HIGHER-CURVATURE GRAVITY AND NON-FACTORIZABLE METRICS}

\section{A. General remarks}

We consider the non-factorizable ansatz (1) for the fivedimensional metric [1,2], which recently attracted a great deal of attention because of its connection with the view of our world as a D(irichlet)-brane embedded in the fivedimensional geometry [3,2]. Our point in this article is to examine first whether such metrics are compatible with the low-energy effective action obtained from the $O\left(\alpha^{\prime}\right)$ string effective action (4). As we shall show below, it is only for a particular (positive) sign of the GB term (8) relative to the Einstein term, which is the case obtained from string amplitudes [8], that the equations of motion in the space-time (1) have a real solution. Moreover, we shall also verify that the specific Randall-Sundrum scenario (2) is a solution of the equations of motion under certain conditions.

Assuming that the metric function $\sigma(z)$ in Eq. (1) and the dilaton fields $\Phi(z)$ are functions only of $z$, we write the equations of motion (10),(11) in the form

$$
\begin{aligned}
0= & \frac{e^{\omega \Phi(z)} v(z)}{2}+\frac{e^{\zeta \Phi(z)} \xi(z)}{2}-6 \sigma^{\prime}(z)^{2}+12 e^{\theta \Phi(z)} \lambda \sigma^{\prime}(z)^{4} \\
& -36 e^{\theta \Phi(z)} \theta \lambda \sigma^{\prime}(z)^{3} \Phi^{\prime}(z)-\frac{2 \Phi^{\prime}(z)^{2}}{3} \\
& +12 e^{\theta \Phi(z)} \theta^{2} \lambda \sigma^{\prime}(z)^{2} \Phi^{\prime}(z)^{2}+\frac{8 e^{\theta \Phi(z)} \lambda \Phi^{\prime}(z)^{4}}{27} \\
& +3 \sigma^{\prime \prime}(z)-12 e^{\theta \Phi(z)} \lambda \sigma^{\prime}(z)^{2} \sigma^{\prime \prime}(z) \\
& +24 e^{\theta \Phi(z)} \theta \lambda \sigma^{\prime}(z) \Phi^{\prime}(z) \sigma^{\prime \prime}(z) \\
& +12 e^{\theta \Phi(z)} \theta \lambda \sigma^{\prime}(z)^{2} \Phi^{\prime \prime}(z) \\
0 & \frac{e^{\zeta \Phi(z)} \xi(z)}{2}-6 \sigma^{\prime}(z)^{2}+12 e^{\theta \Phi(z)} \lambda \sigma^{\prime}(z)^{4} \\
& -48 e^{\theta \Phi(z)} \theta \lambda \sigma^{\prime}(z)^{3} \Phi^{\prime}(z)+\frac{2 \Phi^{\prime}(z)^{2}}{3} \\
& -\frac{8 e^{\theta \Phi(z)} \lambda \Phi^{\prime}(z)^{4}}{9} \\
& \\
& \\
&
\end{aligned}
$$

$$
\begin{aligned}
0= & e^{\omega \Phi(z)} \omega v(z)+e^{\zeta \Phi(z)} \zeta \xi(z)+120 e^{\theta \Phi(z)} \theta \lambda \sigma^{\prime}(z)^{4} \\
& +\frac{8 \Phi^{\prime \prime}(z)}{3}-\frac{32 \sigma^{\prime}(z) \Phi^{\prime}(z)}{3}+\frac{256 e^{\theta \Phi(z)} \lambda \sigma^{\prime}(z) \Phi^{\prime}(z)^{3}}{27} \\
& -\frac{16 e^{\theta \Phi(z)} \theta \lambda \Phi^{\prime}(z)^{4}}{9}-96 e^{\theta \Phi(z)} \theta \lambda \sigma^{\prime}(z)^{2} \sigma^{\prime \prime}(z) \\
& -\frac{64 e^{\theta \Phi(z)} \lambda \Phi^{\prime}(z)^{2} \Phi^{\prime \prime}(z)}{9}
\end{aligned}
$$

Owing to the Bianchi identities, only two of the equations are linearly independent in the bulk. It is straightforward to verify the following relation among the equations:

$$
\begin{aligned}
& 8 \sigma^{\prime}(z) \times[(12)-(13)]-\Phi^{\prime}(z) \times(14)+2 \frac{d}{d z}(13) \\
& =e^{\zeta \Phi(z)} \xi^{\prime}(z)+e^{\omega \Phi(z)} v(z)\left[4 \sigma^{\prime}(z)-\omega \Phi^{\prime}(z)\right]
\end{aligned}
$$

Note that, in order to avoid breaking of Poincare invariance in the bulk space-time, which we assume here [1,2], we must impose

$$
\xi^{\prime}(z)=0
$$

It should also be noted, however, that it is possible to preserve Poincaré invariance in the bulk by including a more general dilaton potential $\xi(\Phi)[6,24]$. In the (heterotic) string-inspired context of interest to us here, such potentials may be generated by string-loop corrections. We shall not discuss this case explicitly here, as it will not affect our qualitative conclusions.

\section{B. Constant dilaton case and the Randall-Sundrum space-time}

We commence our analysis with the case of constant dilaton. In this case, we can set $\Phi=\eta=$ const, and $\Phi^{\prime}=\Phi^{\prime \prime}$ $=0$ in the bulk, but not on the brane, since $\Phi^{\prime}$ can be discontinuous there, as we shall discuss later on. In this case the equations of motion are reduced to

$$
\begin{gathered}
e^{\eta \omega} v \delta(z)=\frac{d}{d z}\left[-6 \sigma^{\prime}(z)+8 e^{\eta \theta} \lambda \sigma^{\prime}(z)^{3}\right] \\
e^{\zeta \eta} \xi(z)-12 \sigma^{\prime}(z)^{2}+24 e^{\eta \theta} \lambda \sigma^{\prime}(z)^{4}=0,
\end{gathered}
$$

implying

$$
-6 \sigma^{\prime}(z)+8 e^{\eta \theta} \lambda \sigma^{\prime}(z)^{3}=c=\mathrm{const}
$$

in the bulk. As a third-degree equation this has always a real solution of the form $\sigma^{\prime}(z)=k_{+}, z>0, \sigma^{\prime}(z)=k_{-}, z<0$.

We now integrate Eq. (17) over $z$ to an interval that includes the brane at $z=0$ :

$$
e^{\eta \omega} v=-6 \sigma^{\prime}(z)+\left.8 e^{\eta \theta} \lambda \sigma^{\prime}(z)^{3}\right|_{0_{-}} ^{0^{+}},
$$

which reduces to 


$$
e^{\eta \omega} v=-6\left(k_{+}-k_{-}\right)+8 e^{\eta \theta} \lambda\left(k_{+}^{3}-k_{-}^{3}\right),
$$

thus relating $k_{+}, k_{-}$with $v$.

Solving Eq. (18) with respect to $\xi$, by requiring continuity of $\xi(z)$ at $z=0$, we obtain

$$
e^{\eta \zeta} \xi=-12 k_{+}^{2}\left(-1+2 e^{\eta \theta} \lambda k_{+}^{2}\right)=-12 k_{-}^{2}\left(-1+2 e^{\eta \theta} \lambda k_{-}^{2}\right),
$$

which has two solutions. The first one is

$$
k_{+}=-k_{-}=k
$$

which is the RS solution [2]. ${ }^{2}$

The second solution is

$$
k_{+}^{2}+k_{-}^{2}=\frac{e^{-\eta \theta}}{2 \lambda}
$$

and exists only for $\lambda>0$, which is the case compatible with string amplitude computations [8].

From the dilaton equation (14) in the bulk, after taking into account Eq. (18), one has

$$
\zeta+2 e^{\eta \theta}(-\zeta+5 \theta) \lambda k_{+}^{2}=\zeta+2 e^{\eta \theta}(-\zeta+5 \theta) \lambda k_{-}^{2}=0,
$$

which, in conjunction with Eq. (22), leads to either

$$
k_{+}=-k_{-}=k \text { and } \zeta+2 e^{\eta \theta}(-\zeta+5 \theta) \lambda k^{2}=0
$$

Or

$$
\zeta=\theta=0 \text { and } k_{+}^{2}+k_{-}^{2}=\frac{e^{-\eta \theta}}{2 \lambda}
$$

Finally, integrating the dilaton equation (14) in the neighborhood of the brane, we obtain

$$
e^{\eta \omega} \omega v=\left.32 e^{\eta \theta} \theta \lambda \sigma^{\prime}(z)^{3}\right|_{0_{-}} ^{0+}=32 e^{\eta \theta} \theta \lambda\left(k_{+}^{3}-k_{-}^{3}\right) .
$$

From Eqs. (22),(26),(28) we thus have

$$
\begin{gathered}
e^{\zeta \eta} \xi=12 k^{2}-24 k^{4} \lambda \\
e^{\omega \eta} v=4 k\left(-3+4 k^{2} \lambda\right) \\
\zeta=\frac{10 k^{2} \theta \lambda}{-1+2 k^{2} \lambda} \\
\omega=\frac{16 k^{2} \theta \lambda}{-3+4 k^{2} \lambda} .
\end{gathered}
$$

Note that the string solution $\zeta=-\theta \quad\left(=\frac{4}{3}\right.$ for 5 -dimensional string theory) is satisfied for

\footnotetext{
${ }^{2}$ Note that the solution with $k_{+}=k_{-}$has a continuous metric function at $z=0$, and hence is not of the RS type.
}

$$
\omega=\frac{2}{3}, \quad v=-\frac{32 k}{3}, \quad \xi=10 k^{2}, \quad k=\frac{1}{2 \sqrt{3 \lambda}}, \quad \lambda=\frac{1}{8 g_{s}^{2}} .
$$

Since $k$ is positive, we observe that the string-effective action yields, in the case of a constant dilaton, the RS scenario [2] in which the bulk spacetime is of anti-de Sitter (in the sense of a cosmological constant $\xi>0$ in our conventions), while the sign of $v$ is opposite to that of $\xi$ (and hence the brane world at $z=0$ has positive tension). We also notice that the solution (33) implies that the sign of the conformal weight $\omega$ is opposite to that of $\theta$, which is expected from generic considerations in string theory [8].

For the second solution (27), one obtains, on account of Eqs. (22) and (28),

$$
\zeta=\theta=\omega=0
$$

and

$$
v=-6 k_{+}+8 \lambda k_{+}^{3}-2 \sqrt{\frac{1}{2 \lambda}-k_{+}^{2}}-8 \lambda k_{+}^{2} \sqrt{\frac{1}{2 \lambda}-k_{+}^{2}}
$$$$
\xi=-12 k_{+}^{2}\left(-1+2 \lambda k_{+}^{2}\right)
$$

$$
k_{-}=-\sqrt{\frac{1}{2 \lambda}-k_{+}^{2}}
$$

Above, we have chosen the negative solution for $k_{-}$to ensure finiteness of the metric at $|z| \rightarrow \infty$. We observe that the bulk spacetime is again of the anti-de Sitter type, for small $\lambda$, where the perturbative string-effective-action approach is valid, while $v$ and $\xi$ come with opposite signs on the brane at $z=0$.

At this point it is natural to enquire whether a vanishing cosmological constant on the brane occurs by an appropriate choice of the (free) parameter $k_{+}$in the solution (37). Indeed, in the case of a single brane (at $z=0$ ) the fourdimensional cosmological constant $(\Omega)$ is given by

$$
\Omega \equiv \int_{-\infty}^{\infty} \sqrt{-g} \xi+v=\frac{k_{-}-k_{+}}{2 k_{-} k_{+}} \xi+v,
$$

which yields the Randall-Sundrum solution, with

$$
k_{+}=-k_{-}=k=\frac{1}{2 \sqrt{\lambda}}
$$

as the unique solution that guarantees the zero cosmological constant in our framework, where higher-curvature corrections have been taken into account.

So far we have concentrated on the case of a single brane, located at $z=0$. The above conclusions are not affected by including more than one branes, as in the approach of [2], which is needed for a solution of the hierarchy problem. Within our framework, despite the small value of $k$ $=\sqrt{2 / 3} g_{s}$, Eq. (33), in units of $\alpha^{\prime}$, this can be achieved by 
placing another brane at $z=r_{0}$, which we assume describes the location of the observable world [2]. As in Ref. [2], $r_{0}$ may be taken (within the classical framework) to be a free parameter, which may be assumed, much larger than the string scale $l_{s}=\sqrt{\alpha^{\prime}}$. In such a case, the mass hierarchy in our world arises from the fact that the value of the determinant in front of the matter Lagrangian on the brane, at $z$ $=r_{0}$, will be suppressed by exponential factors of the generic form $e^{-k r_{0}}$. These can be small for $r_{0} / \sqrt{\alpha^{\prime}}$ sufficiently large.

However, as we shall discuss in the next section, the general solution to the equations of motion for the stringeffective case imply the possibility of a dynamical appearance of a second brane (domain wall) located at a distance, which is determined by the underlying dynamics, and in fact turns out to be infinite. We should mention that similar restrictions on a dynamically induced magnitude of $r_{0}$ may be encountered in the case when quantum (recoil) fluctuations in the D-branes are considered [14]. We reserve discussion of this problem for a forthcoming publication.

\section{Linear dilaton in Randall-Sundrum space-times}

In this subsection we shall examine the simplest possible case of a non-constant dilaton, namely that of a dilaton linear in the bulk dimension $[17,18]$ :

$$
\Phi(z)=Q z+\eta,
$$

with $Q$ constant.

Considering this case may seem well motivated by the proposal on the identification of the bulk coordinate $z$ as a holographic renormalization group parameter [15], in case the bulk space-time is anti-de Sitter, which is known to exhibit special holographic properties [19]. From this point of view, the linear dilaton ansatz, for a metric of the form (1), is suggestive of a more specific situation, namely that of the identification of $z$ with a (space-like) Liouville mode [16] in the five-dimensional context. However, this identification requires some thinking, and is not always possible. Renormalization-group flow in stringy $\sigma$ models is irreversible, due to the loss of information in modes beyond the ultraviolet (world-sheet) cutoff. This implies the presence of a $c$ theorem [20], whose existence for generic bulk spacetimes is not clear at present $[21,22,6]$. We shall not discuss this interpretation further in this article. This will be the topic of a forthcoming publication.

Nevertheless, in this section we shall study the linear dilaton case (40) per se and discuss whether this ansatz is compatible with the metric (1) in the context of the $O\left(\alpha^{\prime}\right)$ string-effective action (4). To this end, we first consider the linear combination $(12)-3 \times(13)$ and substitute the ansatz (40) for the dilaton and the RS metric (2). In such a case, we obtain in the bulk,

$$
\begin{aligned}
& -72 Q^{2}+64 e^{\theta \Phi(z)} Q^{4} \lambda+648 e^{\theta \Phi(z)} k^{3} Q \theta \lambda \\
& +648 e^{\theta \Phi(z)} k^{2} Q^{2} \theta^{2} \lambda .
\end{aligned}
$$

From this, it is trivial to conclude that the linear dilaton solution is compatible only with $\theta=0$, which on account of the equations of motion leads to $\zeta=\omega=0$. In this case, we find two solutions for $\lambda>0$ :

$Q^{2}=\frac{9}{8 \lambda}, \quad k^{2}=\frac{1}{2 \lambda}, \quad \xi=\frac{1}{4 \lambda}, \quad v=-18 \sqrt{\frac{2}{\lambda}}$

or

$Q^{2}=\frac{9}{8 \lambda}, \quad k^{2}=\frac{2+\sqrt{6}}{8 \lambda}, \quad \xi=0, \quad v=3 \sqrt{\frac{52+22 \sqrt{6}}{\lambda}}$

We now remark that, from the point of view of a possible holographic renormalization-group interpretation of the bulk geometry $[15,19]$, the consistent solution would be the first one (42), characterized by an anti-de Sitter type bulk geometry. Because of the $z$ independence of $Q$ in this case, the fixed points connected with the renormalization-group flow (i.e. the theories residing on the two branes in the RS geometry) would be degenerate, being characterized by the same value of the central charge $Q$, and hence would be connected by marginal operators in a RG sense on the world sheet. This case is common in superstring theories.

Note the relative sign difference in $v$ between the two solutions. Also notice that in neither of the above cases is it possible to fine-tune the parameters so as to obtain a vanishing cosmological constant on the four-dimensional world. The cosmological constant is relatively small, for weakly coupled strings, as being proportional to $g_{s}$. However, this is not phenomenologically acceptable, unless one considers (non-realistic) very weakly coupled string theories.

As a final remark, we would like to stress the difference of our scenario from those discussed in Ref. [6]. In our case, in contrast to that discussed in [6], there exists the non-trivial fourth-derivative dilaton term $(\nabla \phi)^{4}$. Its presence is crucial in ensuring (for the $\theta=0$ case) the consistency of the lineardilaton ansatz with the non-factorizable metric case and, moreover, in yielding solutions for $\sigma(z)$ that go beyond the RS scenario.

\section{BEYOND THE RANDALL-SUNDRUM SCENARIO}

In this section we shall examine the general solution, within the string effective action framework, for the spacetime (1), where we shall treat both $\sigma(z)$ and the dilaton $\Phi(z)$ as unknown functions, without restriction to the specific form of the RS metrics (2). We shall discuss the general solution of the equations of motion (12)-(14), and discuss the connection with the metrics (2) as a special case. As we shall demonstrate below, sufficient analytic information on the structure of the solutions can be obtained, which allows us to draw some general conclusions on the underlying physics. 


\section{A. General solution of the graviton and dilaton equations}

It is convenient to use the notation

$$
\begin{gathered}
y(z) \equiv \lambda e^{\theta \Phi(z)}, \quad u(y) \equiv \frac{1}{\sqrt{y}} \frac{d y(z)}{d z}, \\
q \equiv \sqrt{y} \frac{d \sigma(z)}{d z}, \quad \Xi=\xi \lambda^{-\zeta / \theta} .
\end{gathered}
$$

From now on, we shall concentrate on the case of string theory $\zeta=-\theta \quad(=4 / 3$ in the case of five-dimensional strings). In the above parametrization, Eq. (13) becomes algebraic:

$$
16 \Xi-192 q^{2}+384 q^{4}-1536 q^{3} u+12 u^{2}-9 u^{4}=0 .
$$

Solving Eqs. (12),(14) with respect to $q^{\prime}, u^{\prime}$ and using Eq. (45) we obtain

$$
\begin{aligned}
y \frac{d q(y)}{d y} & =\frac{A(q, u)}{8 u C(q, u)} \\
y \frac{d u(y)}{d y} & =\frac{B(q, u)}{u C(q, u)},
\end{aligned}
$$

where

$$
\begin{aligned}
A(q, u)= & -\left(-4 q+48 q^{3}-2 u-16 q^{2} u+u^{3}\right) \\
& \times\left(128 q^{3}-2 u+3 u^{3}\right) \\
B(q, u)= & 4 q\left(1-4 q^{2}+12 q u\right) \\
& \times\left(-4 q+48 q^{3}-2 u-16 q^{2} u+u^{3}\right) \\
C(q, u)= & -2+8 q^{2}-512 q^{4}-16 q u+3 u^{2}-12 q^{2} u^{2} \\
& +24 q u^{3} .
\end{aligned}
$$

Dividing the two equations in Eqs. (46) we obtain

$$
\frac{d q}{d u}=\frac{128 q^{3}-2 u+3 u^{3}}{32 q\left(-1+4 q^{2}-12 q u\right)} .
$$

Notice that the same equation is obtained by simply differentiating the algebraic equation (45) with respect to $u$, thus demonstrating that this equation provides the general solution $q=q(u)$. This is a one-parameter family of solutions, with the parameter being provided by the bulk cosmological constant $\Xi$. This result was to be expected, considering the fact that the three equations are not independent [cf. Eq. (15)]. Using the result for $q(u)$ we can formally solve Eq. (46) for $u(y)$,

$$
y=y_{0} \exp \left(\int d u u \frac{C[q(u), u]}{B[q(u), u]}\right),
$$

from which, on account of Eqs. (46) and (44), we obtain $y(z)(\Phi(z))$ and $\sigma(z)$ as functions of the bulk coordinate $z$. However, in practice the analysis is obscured by the presence of divergences in the derivatives of $(q, u)$, which are re- solved only in the physical parametrization $(\sigma(z), \Phi(z))$. In this case we resort to numerical integration of the full system (12)-(14).

We first note that the above equations admit in the bulk some exact solutions, which are known analytically. The first example is the trivial case of

$$
\Xi=u=q=0,
$$

corresponding to a flat bulk space-time with a constant dilaton, which is obviously an exact solution of the equations of motion.

A second exact solution occurs for anti-de Sitter bulk with a specific value of the cosmological constant:

$$
q^{2}=\frac{1}{12}, \quad u=0, \quad \Xi=\frac{5}{6} .
$$

This is the Randall-Sundrum (constant dilaton) solution (33), derived in Sec. III B.

A third exact solution can be found by inspecting Eqs. (46),(47). We notice that both $d q / d y$ and $d u / d y$ vanish for

$$
\begin{gathered}
q=q_{0}, \quad u=u_{0} \neq 0 \text { with } \\
-4 q_{0}+48 q_{0}^{3}-2 u_{0}-16 q_{0}^{2} u_{0}+u_{0}^{3}=0
\end{gathered}
$$

and thus the above points correspond to exact solutions with $\Xi$ determined from Eq. (45). These solutions correspond to a curve in the phase space (see the discussion in Sec. IV C and Fig. 2). In terms of the metric and the dilaton these solutions are singular:

$$
\sigma(z)=\sigma_{0}+\sigma_{1} \ln \left(z-z_{0}\right), \quad \Phi(z)=-\frac{3}{4} \ln \phi_{0}-\frac{3}{2} \ln \left(z-z_{0}\right),
$$

with $\phi_{0}=1+\sigma_{1} /\left(2-8 \sigma_{1}^{2}+12 \sigma_{1}^{3}\right)$ and $\Xi$ determined from Eq. (45) in the range $0.60<\Xi<44.44$.

Finally, another exact solution is

$$
q=0, \quad u= \pm \sqrt{2}, \quad \Xi=\frac{3}{4}
$$

or, in terms of the original parameters,

$$
\sigma(z)=\text { const }, \quad \Phi(z)=-\frac{3}{2} \ln \left(\frac{z}{\sqrt{2 \lambda}}\right), \quad \xi(z)=\frac{3}{4 \lambda}
$$

which implies a flat bulk space-time, with a non-constant dilaton.

The general solution of Eqs. (46) and (45) is represented by a (Escher-illusion-like [25]) phase-space diagram given in Fig. 1. The shaded region corresponds to de Sitter type bulk, $\Xi<0$, while the rest of the graph corresponds to the case of interest here, namely anti-de Sitter type bulk, $\Xi>0$. The various contours in the diagram of Fig. 1 correspond to solutions with various values of the cosmological constant $\Xi$ $=\lambda \xi$. For instance, the depicted contours in the anti-de Sitter region in the upper-right side of the graph correspond to 


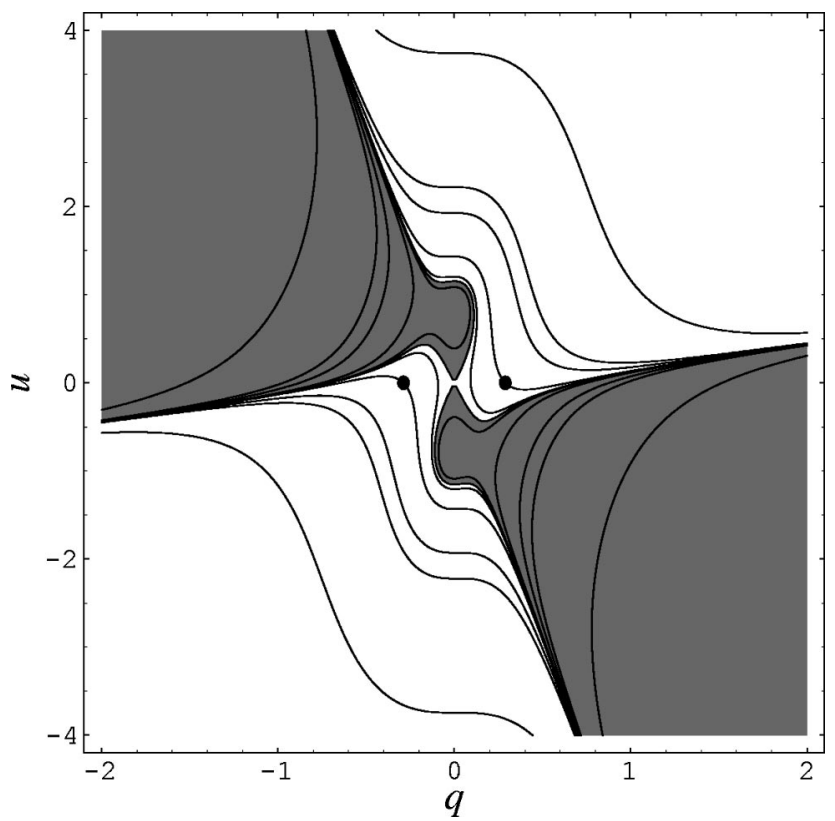

FIG. 1. Phase-space diagram $u(q)$ for the general solution of the five-dimensional equations of motion for dilaton and graviton fields in the presence of $O\left(\alpha^{\prime}\right)$ terms in the string-inspired effective action. The various contours are parametrized by the values of the bulk cosmological constant ( $\Xi$ ). The shaded region corresponds to de Sitter bulk space-times $(\Xi<0)$, while the rest of the diagram corresponds to anti-de Sitter bulk ( $\Xi>0)$. The boundaries between these two regions correspond to the $\Xi=0$ contours. The dots represent the Randall-Sundrum space-time. The origin $(u=q=0)$ corresponds to a flat bulk space-time.

the following values of $\Xi: 0,0.1,5 / 6,5,10,100$, in increasing sequence, pointing outwards from the center of the graph.

The above-discussed exact solutions (50)-(55) correspond to specific points in that diagram. For instance, the trivial flat space-time appears at the origin $(0,0)$ of the solution space, while the RS solution (51) corresponds to the marked points in Fig. 1.

From the graph it becomes clear that there are only four singular points, corresponding to the cases $q \rightarrow \pm \infty, u \rightarrow \pm$, in which $q / u \sim$ const. We shall study these points analytically in the next subsection.

\section{B. Singularity structure in $(u, q)$ parameter space}

In this section we perform an analytic study of the singular points of the solution space of Eqs. (46) in the $(u, q)$ parametrization. It would be instructive to consider first the connection between singularities in the physical parameters $\sigma, \Phi$ and their derivatives and the transformed space parameters $q, u, y$ and their derivatives. One easily concludes from Eqs. (44) that when $q, u$ diverge there are divergences in at least one of the quantities $\Phi, d \sigma / d z, d \Phi / d z .^{3}$

\footnotetext{
${ }^{3}$ The inverse is also true; that is, finite $q$ and $u$ correspond to finite $\sigma, \sigma^{\prime}$ and $\Phi, \Phi^{\prime}$ with the exception of the points of the exact solution (53).
}

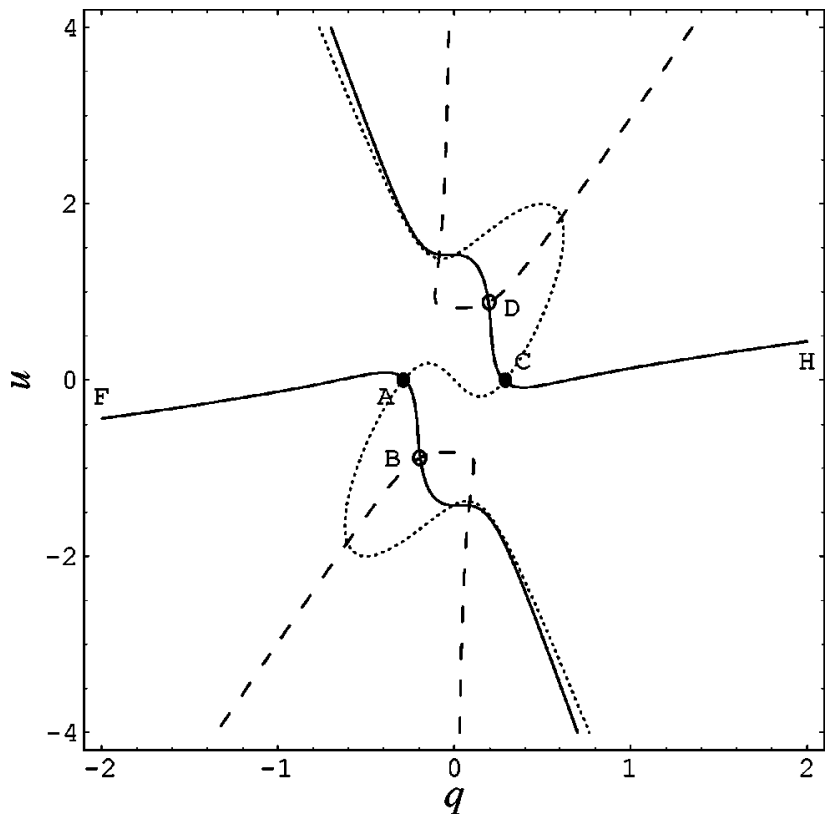

FIG. 2. A solution interpolating between the Randall-Sundrum solution at $z=+\infty$ and a naked singularity [see Eqs. (59) for the case $\rho_{2}$ ] at $z=0$. There are two (physically equivalent) branches $\mathrm{CH}$ and AF. There are other solutions that encounter (D or B) non-resolvable singularities in the derivatives $d q / d y, d u / d y$ (dashed curves). The dotted curve (for $u \neq 0$ ) corresponds to the exact solutions of Eq. (53).

Regarding the second derivatives we have

$$
\frac{d^{2} \sigma}{d z^{2}}=u\left(\frac{d q}{d y}-\frac{q}{2 y}\right), \quad \frac{d^{2} y}{d z^{2}}=u\left(\frac{d u}{d y}+\frac{u}{2 y}\right),
$$

and thus singularities in $d q / d y, d u / d y$, for $u, q, y=$ finite, correspond to singularities in the derivatives of the physical parameters in all cases except $u \rightarrow 0$ with $u(d q / d y)=$ finite and $u(d u / d y)=$ finite.

We are now ready to proceed to a study of the singularities. The right-hand side of Eqs. (46) does not contain any explicit dependence on $y$ and, thus, can be easily examined for singularities at the cases $q \rightarrow \pm \infty$ and/or $u \rightarrow \pm \infty$ and/or $q \rightarrow 0$ and/or $u \rightarrow 0$. After a systematic search (see Fig. 1) of all cases we find only one class of four singular solutions at $q \sim u \rightarrow \pm \infty$ :

$$
q \sim \rho_{i} u, \quad u \sim u_{0} y^{c_{i}}
$$

where $\rho_{i}, i=1,2$ is one of the real solutions of the equation $-3-512 \rho^{3}+128 \rho^{4}=0, \rho_{1} \approx-0.178, \rho_{2} \approx 4.000$ and

$$
c_{i}=\frac{1-16 \rho_{i}^{2}+48 \rho_{i}^{3}}{32\left(-3+\rho_{i}\right) \rho_{i}^{2}},
$$

$c_{1} \approx-0.070, c_{2} \approx 5.500$.

We thus conclude [cf. Eqs. (44)] that the singularities in the solution space are encountered at $y \rightarrow 0 \quad(\Phi \rightarrow+\infty)$ for $\rho_{1}$ and $y \rightarrow+\infty(\Phi \rightarrow-\infty)$ for $\rho_{2}$. From Eqs. (44) one ob- 
serves that, near the singular points, the dilaton $y[\Phi(z)]$ and metric $\sigma(z)$ functions acquire the form

$$
\begin{aligned}
y^{1 / 2-c_{i}} & =\left(\frac{1}{2}-c_{i}\right) u_{0} z>0, \quad y(z)=\left[u_{0}\left(\frac{1}{2}-c_{i}\right) z\right]^{1 /\left(1 / 2-c_{i}\right)}, \\
\Phi(z) & =\Phi_{0}-\frac{3}{2-4 c_{i}} \ln |z|, \\
\sigma(z) & =\sigma_{0}+\frac{\rho_{i}}{\frac{1}{2}-c_{i}} \ln |z|
\end{aligned}
$$

where $\sigma_{0}, u_{0}, \Phi_{0}=$ const. Several remarks are in order at this point: (i) Both singularities occur at $z=0$, but we have the condition $u_{0} z>0$ (for $\rho_{1}$ ), and $u_{0} z<0$ (for $\rho_{2}$ ), so if one assumes a fixed sign of $u_{0}$ for both singularities, then we see that one approaches $z=0$ from different side for each type of singular solution. (ii) As one approaches the singularities, both the dilaton and metric functions diverge logarithmically with $z$. (iii) The scalar curvature in the five-dimensional bulk near the singularity is given by

$$
R=4\left\{5\left[\sigma^{\prime}(z)\right]^{2}-2 \sigma^{\prime \prime}(z)\right\}=4(5 \gamma-2) \gamma \frac{1}{z^{2}}, \quad \gamma=\frac{\rho_{i}}{\frac{1}{2}-c_{i}}
$$

We observe from Eqs. (60) that the curvature diverges as $z$ $\rightarrow 0$, and thus one has a naked singularity there. However, for a four-dimensional observer, residing on the brane at $z$ $\rightarrow 0$, the singularity for both cases is integrable, given that the (covariant) integral of the scalar curvature over $z$ in the vicinity of the singularity yields

$$
\int d z \sqrt{g} R(z) \propto \int_{z \sim \epsilon \rightarrow 0} d z z^{-4\left(\gamma_{i}+1 / 2\right)} \sim \epsilon^{-4 \gamma_{i}-1} \rightarrow 0,
$$

since the exponent $-4 \gamma_{i}-1$ takes on the positive values 0.25 and 2.2 for the $\rho_{1}$ and $\rho_{2}$ cases respectively.

By closely inspecting the general solution we observe (see Fig. 1 and for a detailed view Fig. 2) that the RS solution (51) is not isolated, but is connected by means of a continuous interpolating function with the naked singularity $\rho_{2}$, Eq. (59). The important issue is to determine the point in the $z$ axis to which the RS solution corresponds. This will be the topic of the next subsection.

Before doing so we should remark that there are other branches of the solution space that connect the RS solution with genuine singularities of the derivatives of $q$ and $u$ at points in which the $C(q, u)$ factor in the denominator of Eqs. (46) vanishes, for $q, u$ finite and nonzero. This becomes evident from Fig. 2 where we plot the contours crossing the RS points $(A, C)$ as well as the curves (dashed lines) representing the above (non-resolvable) divergences in the derivatives $d q / d y, d u / d y$. Such points may correspond to naked singu- larities in the $\sigma(z)$ and $\Phi(z)$ space, given that the first and second derivatives of both fields diverge, assuming finite values of $y$.

We have also plotted in Fig. 2 the curve that represents the (one parameter) exact solution of Eq. (53) (dotted curve). The various points on the curve correspond to exact solutions with different values of $\Xi$. The curve does not contain the points $A, B$, since the $u=0$ points are excluded.

In this article we shall not discuss these branches of the general solution further. A detailed study is postponed to a future publication.

\section{Interpolating between the Randall-Sundrum solution and naked singularities}

Let us now proceed to an analytic determination of the behavior of the solution in the neighborhood of the RS points ( $A, C$ in Fig. 2), which will also determine the point in the $z$ axis to which the RS solution corresponds.

Expanding (45) around $q=q_{0}= \pm 1 / \sqrt{12}, u=0$ we obtain $q \sim q_{0}-\frac{1}{2} u$, which on account of Eqs. (46),(47) leads to

$$
\begin{aligned}
& q= \pm \frac{1}{2 \sqrt{3}} \mp \frac{1}{\sqrt{3}} \ln \frac{y}{y_{0}}, \\
& u= \pm \frac{2}{\sqrt{3}} \ln \frac{y}{y_{0}}
\end{aligned}
$$

with $y \rightarrow y_{0}=$ finite. The sign of $y-y_{0}$ determines the branch of the solutions depicted in Fig. 2. For the $A F$ or $\mathrm{CH}$ branches, which we shall study here, $y<y_{0}$, on account of Eqs. (62) (the opposite is true for the $A B, C D$ branches).

From Eqs. (44) we have

$$
y=y_{0}-e^{\left( \pm 2 / \sqrt{3} y_{0}\right) z},
$$

which implies that the point $y \rightarrow y_{0}$ occurs at $z \rightarrow \mp \infty$, for $y_{0}$ finite and $q_{0}= \pm \sqrt{1 / 12}$ respectively.

Solving for the dilaton $\Phi(z)$ and metric $\sigma(z)$ functions, we then obtain

$$
\begin{aligned}
& \Phi(z) \simeq \Phi_{0}+\frac{3}{4 y_{0}} e^{\left( \pm 2 / \sqrt{3 y_{0}}\right) z}+\cdots, \\
& \sigma(z)=\sigma_{0} \pm \frac{1}{\sqrt{12 y_{0}}} z+\frac{1}{8 y_{0}} e^{\left( \pm 2 / \sqrt{3 y_{0}}\right) z}+\cdots,
\end{aligned}
$$

where $\Phi_{0} \equiv-\frac{3}{4} \ln \left(y_{0} / \lambda\right)$.

Thus, we see that the leading parts of the solution (64), for infinite $z$ (e.g., $z \rightarrow+\infty$ for $q_{0}=-1 / \sqrt{12}$ ), is a smooth Randall-Sundrum type (with $k=-1 / \sqrt{12 y_{0}}$ ), which should be understood only as the part of the RS metric (2) inside a given region of the bulk space-time, bounded by a membrane located at a position $z \rightarrow \infty$ in the bulk. In fact the solution is valid only near the membrane wall, and deviations from it are exponentially suppressed with $z$. The reader should not be alarmed by the apparent divergent form of the metric 

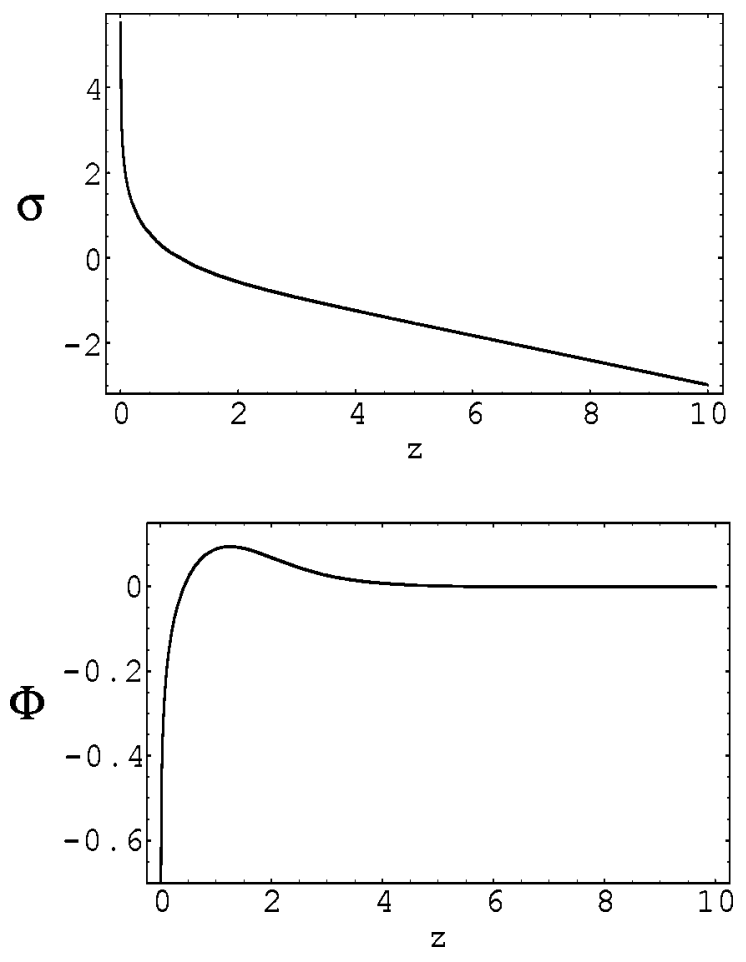

FIG. 3. The metric $\sigma(z)$ and dilaton $\Phi(z)$ as functions of $z$ interpolating between a RS type solution $\left(q_{0}=-\sqrt{1 / 12}\right)$ at $z=+\infty$ and a naked singularity [see Eqs. (59) for $\rho_{2}$ ] at $z=0$. The existence of this solution implies the dynamical restriction of the bulk space-time to the positive $z$ axis.

element as $z \rightarrow \infty$. The correct way of viewing Eqs. (64) is to consider first the solution as valid for $z=\Lambda$, where $\Lambda$ is larger than any other length scale in the problem. Then, one may shift $z \rightarrow \tilde{z}=\Lambda-z$, and arrange the constant $\sigma_{0}$ of Eqs. (64) to be such as to cancel factors of $\left(q_{0} / \sqrt{y_{0}}\right) \Lambda$. Eventually, one may take the limit $\Lambda \rightarrow \infty$. The resulting metric is of the RS type around $\widetilde{z}=0$, while the naked singularity now occurs at $\tilde{z}=\infty$.

At this point we should also remark that inspection of the phase-space diagram of Fig. 1 reveals that the interpolation of the RS solution passes through the point $u=0$ twice. In the journey from $z=+\infty$ towards finite values, the solution passes first through another point $z_{0}>0$ that has $u=0$, before reaching the naked singularity $\rho_{2}$, Eq. (59), at $z=0$. In the point $z=z_{0}$ the behavior of both $\Phi(z)$ and $\sigma(z)$ functions is perfectly regular. Indeed, this second point of vanishing $u$ occurs for $q \rightarrow q_{1}= \pm \sqrt{5 / 12}$. Expanding around this point, one obtains $z-z_{0}=O\left(\left(y-y_{1}\right)^{1 / 2}\right)$, for $y \rightarrow y_{1}$, where $z_{0}>0$ is finite. From Eqs. (44), then, it is evident that, for $z \sim z_{0}$, $\Phi(z)=\Phi_{1}-O\left(z^{2}\right)$ and $\sigma(z)=$ const $+O(z)$, where $\Phi_{1}$ $=(1 / \theta) \ln \left(y_{1} / \lambda\right)$ is a constant. This is a perfectly regular behavior in the $z$ space.

The numerical analysis summarized in Fig. 2 indicates that there exist smooth functions for $\Phi(z)$ and $\sigma(z)$ interpolating between the RS solution at $z=+\infty$ and the naked (integrable) $\rho_{2}$ singularity (59) at $z=0$. These are plotted in Fig. 3 for the case $q_{0}=-\sqrt{1 / 12}$.

The existence of the interpolating solution depicted in
Fig. 3 implies an important fact about the nature of the solution in the context of the string-effective action in which it was derived. The induced bulk space-time is dynamically restricted on the positive $z$ axis (for definiteness, if one considers the $q=-\sqrt{1 / 12}$ branch, which corresponds to the contour segment AF in Fig. 2). In this scenario, our (flat) four dimensional world is viewed as the boundary of the anti-de Sitter bulk ( $\Xi=5 / 6)$ located at $z=\infty$. A RS-type solution is valid near our world, which however deviates from it as $z$ runs towards the origin $z=0$, to become an integrable naked singularity there.

In this scenario, we observe that the dynamics of the $O\left(\alpha^{\prime}\right)$ perturbative string theory yielded important information on the structure of the bulk space-time, which may be related with solitonic (non-perturbative) structures such as D-branes. The non-perturbative nature of the solution we have thus obtained becomes clear from the fact that in terms of the original parameters of the model, the $k$ conformal parameter of the RS solution (51) is found proportional to $1 / \sqrt{\lambda} \sim g_{s}$, where $g_{s}$ is the string coupling.

From the point of view of a holographic RG interpretation of the bulk coordinate $(z)$, we remark that the solution of Fig. 3 satisfies a " $c$ theorem" in the sense of Ref. [21]. Namely, we observe that $\sigma^{\prime \prime}(z)>0$ for $0<z<\infty$, which implies that the weakest energy condition is satisfied for this portion of the bulk space-time.

\section{CONCLUSIONS AND OUTLOOK}

In the present article we have performed a systematic study of non-factorizable metrics of the form (1) in the specific case of five-dimensional geometries. We have considered the situation in which such geometries are derived as consistent solutions of the equations of motion of string effective actions in the five-dimensional case, to $O\left(\alpha^{\prime}\right)$ in the Regge slope. Such terms include quadratic-curvature contributions of the Gauss-Bonnet type, as well as fourth-order dilaton derivative terms.

Our analysis has shown that it is indeed possible to find compatibility of such a string-inspired model with the Randall-Sundrum scenario, upon the appropriate embedding of three branes in the five-dimensional space-time. In addition, we were able to find more general situations, which interpolate between the RS metric at the boundary of an anti-de Sitter bulk and an (integrable) naked singularity at the origin. Such scenarios imply the dynamical formation of domain walls in the space-time, which may be useful when one discusses the consistent embedding of $\mathrm{D}$ (irichlet)-branes in such a picture (as is the case of the original RS scenario). In our solutions the conformal parameter $k$ of the RS type metric, as well as the bulk cosmological constant, turns out to be proportional to the string coupling.

There are many issues that remain to be checked: first the stability of the solution against the inclusion of higher order $\alpha^{\prime 2}$ corrections as well as string-loop corrections. Moreover, in the present work we have assumed that the dilaton and metric functions depend only on the bulk coordinate, and we took the four-dimensional world to be flat. The extension to more complicated metrics, especially time dependent, is 
needed in order to discuss cosmological implications $[9,6,26]$. Moreover, the proper inclusion of quantum fluctuating (recoiling) D-branes, in the way discussed in [14], a situation that undoubtedly is expected to be encountered in a complete quantum theory, is a very interesting issue that deserves special attention and is currently under investigation.

In addition, the precise connection of the bulk coordinate with a holographic renormalization-group parameter in the case of anti-de Sitter bulk geometries also merits a separate study. As mentioned in the text, one should re-examine carefully this interpretation in the context of the existence of a proper $c$ theorem, expressing the irreversibility of the renormalization group flow in the bulk. In Ref. [21], this $c$ theorem was suggested to be provided by the monotonicity of $\sigma^{\prime}(z)\left[\sigma^{\prime \prime}(z) \geqslant 0\right]$ in the metrics (1), which is the result of a positive energy theorem for consistent matter to be placed in the bulk. For the interpolating solution of Fig. 3 this has been shown to be valid. However, this is not always true $[6,22]$ for generic bulk (anti-de Sitter) geometries, especially in the higher-curvature context discussed here, where the presence of the Gauss-Bonnet terms complicates the positive energy conditions [11]. A detailed study of such issues will appear in a forthcoming publication.

\section{ACKNOWLEDGMENTS}

The work is partially supported by the European Union (contracts ERBFMRX-CT96-0090, HPRN-CT-2000-00152). The work of N.E.M. is also partially supported by PPARC (UK).
[1] V. A. Rubakov and M. E. Shaposhnikov, Phys. Lett. 125B, 136 (1983); 125B, 136 (1986); K. Akama, in Gauge Theory and Gravitation, edited by K. Kikkawa, N. Nakanishi and H. Nariai (Springer-Verlag, Berlin, 1983); M. Visser, Phys. Lett. 159B, 22 (1985); M. Gogberashvili, Mod. Phys. Lett. A 14, 2025 (1999).

[2] L. Randall and R. Sundrum, Phys. Rev. Lett. 83, 3370 (1999); 83, 4690 (1999).

[3] N. Arkani-Hamed, S. Dimopoulos, and G. Dvali, Phys. Lett. B 429, 263 (1998); I. Antoniadis, N. Arkani-Hamed, S. Dimopoulos, and G. Dvali, ibid. 436, 257 (1998).

[4] S. Kachru, M. Schulz, and E. Silverstein, Phys. Rev. D 62, 045021 (2000).

[5] C. Csaki, J. Erlich, C. Grojean, and T. Hollowood, Nucl. Phys. B584, 359 (2000); P. Binétruy, J. M. Cline, and C. Grojean, hep-th/0007029.

[6] J. E. Kim, B. Kyae, and H. M. Lee, Nucl. Phys. B582, 296 (2000); I. Low and A. Zee, hep-th/0004124; S. Nojiri and S. D. Odintsov, J. High Energy Phys. 07, 049 (2000).

[7] B. Zwiebach, Phys. Lett. 156B, 315 (1985).

[8] E. S. Fradkin and A. A. Tseytlin, Nucl. Phys. B262, 1 (1985); D. J. Gross and J. H. Sloan, ibid. B291, 41 (1987); R. R. Metsaev and A. A. Tseytlin, ibid. B293, 385 (1987).

[9] I. Antoniadis, J. Rizos, and K. Tamvakis, Nucl. Phys. B415, 497 (1994)

[10] R. Easther and K. Maeda, Phys. Rev. D 54, 7252 (1996).

[11] P. Kanti, N. E. Mavromatos, J. Rizos, K. Tamvakis, and E. Winstanley, Phys. Rev. D 54, 5049 (1996).

[12] S. O. Alexeyev and M. V. Pomazanov, Phys. Rev. D 55, 2110 (1997).

[13] A. Campbell-Smith and N. E. Mavromatos, Phys. Lett. B 476, 149 (2000); 488, 199 (2000).

[14] G. Leontaris and N. E. Mavromatos, Phys. Rev. D 61, 124004 (2000).
[15] S. S. Gubser, I. R. Klebanov, and A. M. Polyakov, Phys. Lett. B 428, 105 (1998); E. Alvarez and C. Gomez, hep-th/9810102; J. de Boer, E. Verlinde, and H. Verlinde, J. High Energy Phys. 08, 003 (2000); E. Verlinde and H. Verlinde, ibid. 05, 034 (2000), and references therein; A. Dhar and S. R. Wadia, hep-th/0006043.

[16] John Ellis, N. E. Mavromatos, and D. V. Nanopoulos, “Erice SubNuclear Series,"' 1993, hep-th/9403133, Vol. 31, p. 1; J. Chaos, Solitons Fractals 10, 345 (1999); John Ellis and N. E. Mavromatos, Int. J. Mod. Phys. A 14, 3761 (1999), and references therein.

[17] F. David, Mod. Phys. Lett. A 3, 1651 (1988); J. Distler and H. Kawai, Nucl. Phys. B321, 509 (1989).

[18] I. Antoniadis, C. Bachas, J. Ellis, and D. V. Nanopoulos, Phys. Lett. B 211, 393 (1988); Nucl. Phys. B328, 117 (1989).

[19] J. Maldacena, Adv. Theor. Math. Phys. 2, 231 (1998); E. Witten, ibid. 2, 253 (1998).

[20] A. B. Zamolodchikov, Pis'ma Zh. Éksp. Teor. Fiz. 43, 565 (1986) [JETP Lett. 43, 730 (1986)]; Yad. Fiz. 46, 1819 (1987) [Sov. J. Nucl. Phys. 46, 1090 (1987)].

[21] D. Z. Freedman, S. S. Gubser, K. Pilch, and N. P. Warner, hep-th/9904017.

[22] For a critical review see E. Witten, hep-ph/0002297, and references therein.

[23] B. de Witt, 'Dynamical Theory of Groups and Fields,' lectures given at the University of North Carolina, 1963 (unpublished).

[24] O. DeWolfe, D. Z. Freedman, S. S. Gubser, and A. Karch, Phys. Rev. D 62, 046008 (2000); G. T. Horowitz, I. Low, and A. Zee, ibid. 62, 086005 (2000).

[25] See M.C. Escher, “Liberation,'” lithograph, 1955, for instance at http://www.WorldOfEscher.com/gallery/Liberation.html

[26] M. Brändle, A. Lukas, and B. A. Ovrut, Phys. Rev. D (to be published), hep-th/0003256. 